Article

\title{
First Evaluation of a New Dynamic Scoring System Intended to Support Prescription of Adjuvant CytoSorb Hemoadsorption Therapy in Patients with Septic Shock
}

\author{
Klaus Kogelmann ${ }^{1, * \mathbb{D}}$, Tobias Hübner ${ }^{2} \mathbb{D}$, Franz Schwameis ${ }^{3}$, Matthias Drüner ${ }^{1}$, Morten Scheller ${ }^{1}$ \\ and Dominik Jarczak 4 (D) \\ 1 Department of Anesthesiology and Intensive Care Medicine, Klinikum Emden, Bolardusstr. 20, \\ 26721 Emden, Germany; m.druener@klinikum-emden.de (M.D.); m.scheller@klinikum-emden.de (M.S.) \\ 2 Department of Anesthesiology and Intensive Care, Kantonsspital Münsterlingen, Spitalcampus 1, \\ 8596 Münsterlingen, Switzerland; tobias.huebner@stgag.ch \\ 3 Department of Anesthesiology and Intensive Care, Landesklinikum Baden-Mödling, \\ Sr. M. Restituta-Gasse 12, 2340 Mödling, Austria; office@schwameis.com \\ 4 Department of Intensive Care, Universitätsklinikum Hamburg Eppendorf, Martinistr. 52, \\ 20251 Hamburg, Germany; d.jarczak@uke.de \\ * Correspondence: k.kogelmann@klinikum-emden.de; Tel.: +49-(0)-4921-98-3508
}

\section{check for} updates

Citation: Kogelmann, K.; Hübner, T.; Schwameis, F.; Drüner, M.; Scheller, M.; Jarczak, D. First Evaluation of a New Dynamic Scoring System Intended to Support Prescription of Adjuvant CytoSorb Hemoadsorption Therapy in Patients with Septic Shock J. Clin. Med. 2021, 10, 2939. https:// doi.org/10.3390/jcm10132939

Academic Editors: Andrew Bentley and Emmanuel Andrès

Received: 1 June 2021

Accepted: 28 June 2021

Published: 30 June 2021

Publisher's Note: MDPI stays neutral with regard to jurisdictional claims in published maps and institutional affiliations.

Copyright: (c) 2021 by the authors. Licensee MDPI, Basel, Switzerland. This article is an open access article distributed under the terms and conditions of the Creative Commons Attribution (CC BY) license (https:// creativecommons.org/licenses/by/ $4.0 /)$.
Abstract: Introduction: Despite advances in critical care medicine, adjunctive approaches in sepsis therapy have failed to prove their efficacy. Notwithstanding promising results using hemoadsorption (CytoSorb), questions remain concerning timing and dosing. We created a dynamic scoring system (DSS) to assess patients with early septic shock and performed a first evaluation of the system in this patient population. Methods: Data from 502 patients with septic shock according to Sepsis-3 criteria were retrospectively analyzed. Score parameters were documented at the time of diagnosis $\left(\mathrm{T}_{0}\right)$ and $6 \mathrm{~h}$ later $\left(\mathrm{T}_{6}\right)$ to calculate a dynamic score. Survival on day 7 and 56 as well as ICU and hospital mortality were analyzed in regard to the score as well as the delay of hemoadsorption therapy. Results: Of the 502 patients analyzed, 198 received adjunctive CytoSorb treatment and 304 received standard therapy. Septic shock was typically represented by 5 points, while $>6$ points indicated a situation refractory to standard therapy with the worst outcome in patients shown by $>8$ points. The differences in mortality between the score groups $(<6,6-8,>8$ points) were significant. Analysis further showed a significant 56-day, ICU and hospital survival advantage in CytoSorb patients when therapy was started early. Conclusion: We created a scoring system allowing for the assessment of the clinical development of patients in the early phase of septic shock. Applying this approach, we were able to detect populations with a distinct mortality pattern. The data also showed that an early start of CytoSorb therapy was associated with significantly improved survival. As a next step, this easy-to-apply scoring system would require validation in a prospective manner to learn whether patients to be treated with hemoadsorption therapy in the course of septic shock could thereby be identified.

Keywords: inflammation; septic shock; CytoSorb; hemoadsorption; scoring

\section{Background}

Sepsis represents a major challenge for medicine and a significant public health concern [1]. Despite all medical advances in recent years, it continues to be a substantial problem, as to date therapeutic approaches have failed to prove efficacy [2]. Sepsis has major importance from a medical and from an economical viewpoint. Approaches that could contribute to its successful treatment need to be further explored [3].

In recent years, hemoadsorption (CytoSorb) has been used more and more frequently to treat septic shock, especially in refractory conditions, in which standard therapy did not seem to be sufficient enough $[4,5]$. The exact state of 'refractory shock', however, is 
not well defined [6]. As there are several lines of evidence showing that an early start of hemoadsorption therapy might be beneficial $[4,5,7,8]$, start of such an approach should not be delayed too long to preserve chances of success. The primary clinical effect of CytoSorb therapy is reported to be a stabilization in hemodynamics accompanied by the opportunity to decrease catecholamine dosages [4-7]. This has been shown to go along with a concomitant restoration in metabolic parameters $[9,10]$. It therefore appears reasonable to also use hemodynamic (and metabolic) parameters in order to define better criteria for therapy initiation but also to shed more light on the definition of 'refractory shock'. For this reason, a dynamic scoring system (DSS) was created based on established, clinically well-available and hemodynamics-associated parameters such as lactate, volume and catecholamine therapy and their changes within the first $6 \mathrm{~h}$. This system should allow for the assessment of the early phase of septic shock to better define refractory states and finally - to be completed in upcoming, prospective analysis still to be performed-help to identify patients with refractory septic shock, who might benefit most from adjuvant therapy with CytoSorb hemoadsorption, early. As a first step, the dynamic system was evaluated via retrospective data analysis of 502 patients with septic shock, 198 of which had been treated with adjunctive hemoadsorption therapy. Additionally, the impact of therapy delay in regard to the initiation of hemoadsorption was evaluated.

\section{Material and Methods}

\subsection{Ethics Approval, Legal Considerations}

This study was approved by the ethics committee of the General Medical Council of Lower Saxony (reference number Bo/29/2019). The study was carried out according to the Declaration of Helsinki and in accordance with the Good Clinical Practice Protocol (GCP) (2001/20/EEC, CPMP/ICH/135/95), the established standard operating procedures and the local laws and regulations applicable to each country. The study was registered at ClinicalTrials.gov, 6 June 2019 (NCT03977688).

\subsection{Study Design}

This study was a retrospective data analysis in 502 critically ill adult patients. Included were data from 4 interdisciplinary intensive care units (ICU) with comparable procedures (Emden/Germany, Münsterlingen/Switzerland, UKE Hamburg/Germany, Baden-Moedling/Austria). Inclusion criteria comprised coded diagnosis of septic shock (Sepsis-3 criteria) [1]. Septic shock is defined according to the SCCM/ESICM Sepsis-3 definition [1], i.e., vasopressor requirement to maintain a mean arterial pressure of $65 \mathrm{mmHg}$ and serum lactate level $>2 \mathrm{mmol} / \mathrm{L}$ in the absence of hypovolemia. We excluded patients where data records were unavailable for analysis, patients not treated in the ICU and patients where norepinephrine (NE) requirement or lactate were not documented.

\subsection{Objectives}

Survival on day 56 in regard to DSS score was defined as the primary objective. Secondary objectives included survival on day 7, ICU and hospital mortality, as well as the timing of hemoadsorption therapy, catecholamine demand, lactate, inflammatory parameters (PCT, CRP), creatinine, duration of organ support (ventilation, renal replacement therapy (RRT), CytoSorb therapy) and length of stay in ICU and hospital.

\subsection{Assessed Parameters}

The following parameters were assessed: medical history, patient characteristics, disease severity scores (Acute Physiology and Chronic Health Evaluation II-APACHE II, Simplified Acute Physiology Score 2-SAPS 2), hemodynamics (catecholamine demand, heart rate, blood pressure), laboratory parameters (lactate clearance, inflammatory parameters, creatinine), initial volume requirement to achieve intravascular normovolemia, use of either hydrocortisone or a second catecholamine (or both), CytoSorb-therapy specific data (therapy delay after diagnosis of septic shock), duration of organ support (duration of 
mechanical ventilation, renal replacement therapy and CytoSorb therapy), outcome data (day 7 and day 56 survival, ICU and hospital stay and survival) as well as safety relevant issues (adverse events).

\subsection{Data Collection}

Data were stored in the hospital information system and could only be accessed by the investigator in charge. Participating investigators provided their data pseudonymized in a tabular format. Centralized data processing was performed at the Department of Anesthesiology and Intensive Care at Emden Hospital.

\subsection{Procedure}

Collected data were entered into a data matrix, presenting the created dynamic scoring system (Figure 1). Each parameter at the time of septic shock diagnosis $\left(T_{0 h}\right)$ and $6 \mathrm{~h}\left(\mathrm{~T}_{6 \mathrm{~h}}\right)$ later was documented to analyze the initial status as well as the dynamic process in early septic shock. We decided to use the interval of the first $6 \mathrm{~h}$ for our dynamic scoring, as this initial course, which is also targeted by the Sepsis Bundles [11], might play an even more important role than changes over several days. The threshold values are based on Sepsis-3 criteria of septic shock [1] (lactate level $>2 \mathrm{mmol} / \mathrm{L}$ ), the administration of norepinephrine according to the Sequential Organ Failure Assessment (SOFA) Score [12] (norepinephrine $>0.1 \mu \mathrm{g} / \mathrm{kg} / \mathrm{min}$ ) and volume requirement according to the Surviving Sepsis Guidelines [11] (bolus $30 \mathrm{~mL} / \mathrm{kg}$ body weight).

\section{Dynamic Scoring System (DSS) to track early evolution of septic shock}

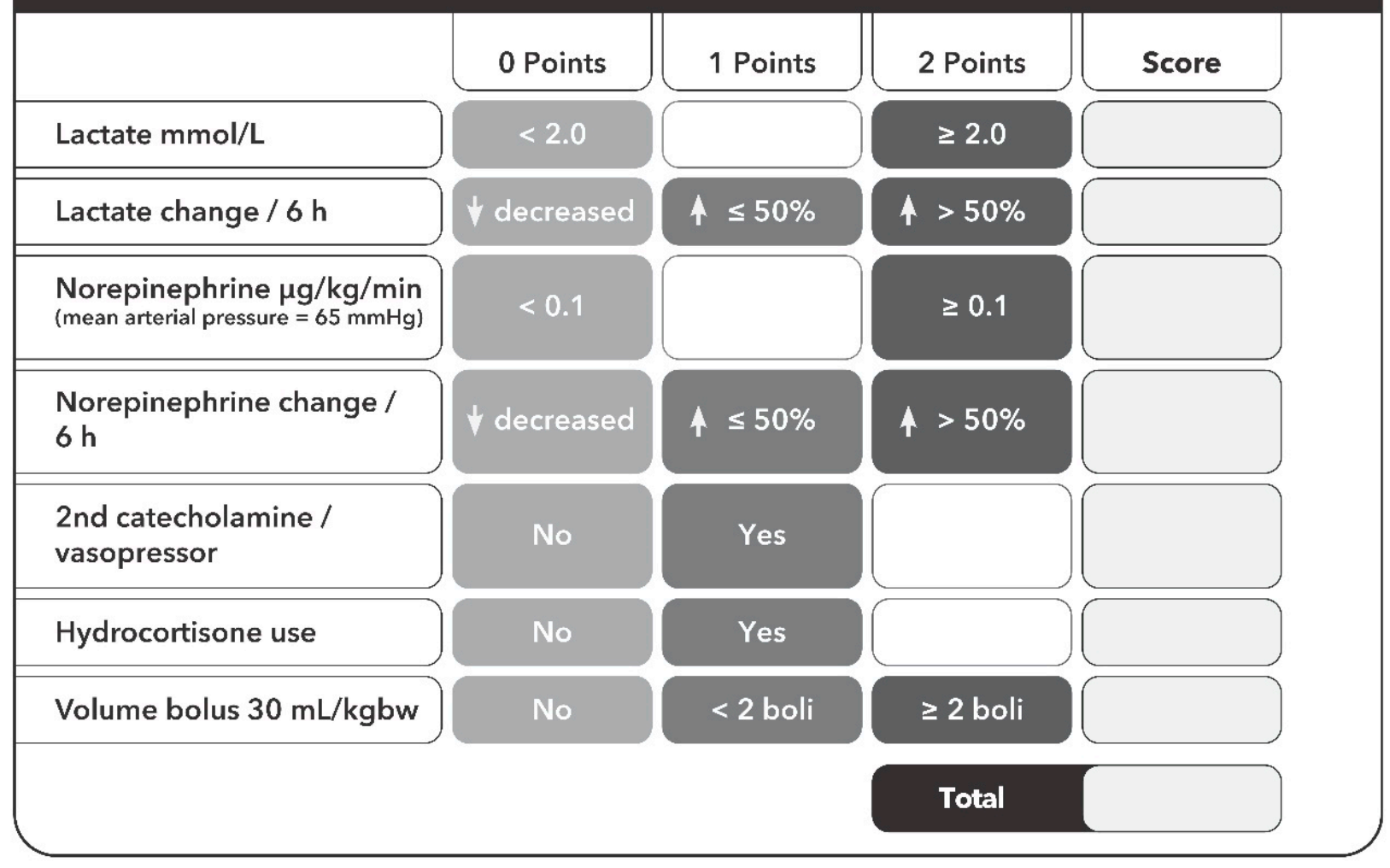

Figure 1. Dynamic Scoring System. Each parameter at the time of septic shock diagnosis $\left(\mathrm{T}_{0 \mathrm{~h}}\right)$ and $6 \mathrm{~h}\left(\mathrm{~T}_{6 \mathrm{~h}}\right)$ later is documented to analyze the dynamic process in early septic shock. Each pathological value is rated with 2 points. Decreasing values or no change during the $6 \mathrm{~h}$ observation period receives no points, increasing values receive 1 point, increasing values $>50 \%$ of the initial value receive 2 points. If $<2$ volume boluses of $30 \mathrm{~mL} / \mathrm{kg}$ are necessary, this is rated with 1 point, when $\geq 2$ volume boluses of $30 \mathrm{~mL} / \mathrm{kg}$ are necessary within $6 \mathrm{~h}$, this is scored with 2 points. 
Each pathological value at time of diagnosis $\left(\mathrm{T}_{\mathrm{oh}}\right)$ was rated with 2 points. If $<2$ volume boluses of $30 \mathrm{~mL} / \mathrm{kg}$ were necessary, this was rated with 1 point, whereas $\geq 2$ volume boluses of $30 \mathrm{~mL} / \mathrm{kg}$ within $6 \mathrm{~h}$ were then scored with 2 points. Further changes after $6 \mathrm{~h}$ $\left(\mathrm{T}_{6 \mathrm{~h}}\right)$ were rated as follows: decreasing values or no change during the observation period received no points, increasing values received 1 point and increasing values $>50 \%$ received 2 points. (Figure 1). Using these data, we finally defined 3 different groups of patients (<6 points, $6-8$ points, $>8$ points). Septic shock was represented by a score of 5 points (lactate $\geq 2 \mathrm{mmol} / \mathrm{L}, \mathrm{NE} \geq 0.1 \mu \mathrm{g} / \mathrm{kg} / \mathrm{min}$ needed to maintain a mean arterial pressure (MAP) $\geq 65 \mathrm{mmHg}$ and initial volume bonus of $30 \mathrm{~mL} / \mathrm{kg}$ applied), while 6 or more points indicated a situation refractory to standard therapy with an even worse clinical course given in patients with $>8$ points.

\subsection{Statistics}

All primary and secondary variables were first examined using an exploratory data analysis method and recorded descriptively. Data are reported as mean \pm standard deviation, frequency and percentage, or median as required. A normal distribution was tested using the Shapiro-Wilk test. Differences in the primary endpoint between study groups were analyzed using the Chi-square $\left(\chi^{2}\right)$ test, using a Bonferroni correction for multiple comparisons $(\alpha /$ number of groups compared $=$ adjusted critical value). To compare the survival function between cohorts, non-parametric Kaplan-Meyer survival analysis was performed on day 7 and 56, estimating and plotting the survival probability as a function of time. The different survival probabilities of groups were then compared to each other regarding statistical significance using a Log-rank test to compare groups. Secondary endpoints were tested with an independent sample $t$-test, or $\chi^{2}$ test, with Bonferroni correction, as required. For non-normal distribution results, nonparametric tests were performed. Data were analyzed with SPSS 20.0, a value of $p<0.05$ was defined as the $\alpha$ (alpha) critical value (statistically significant).

\section{Results}

In the study, 502 patients were included, $61.8 \%$ of whom were male. A total of 198 patients were treated with CytoSorb (39.4\%) and 304 received therapy without CytoSorb (60.6\%). Diagnoses in the study population included pneumonia $(n=219,43.6 \%)$, abdominal sepsis $(n=186,37.1 \%)$, uro sepsis $(n=38,7.6 \%)$ and miscellaneous $(n=59,11.7 \%)$. The baseline characteristics are depicted in Table 1 . With regard to gender, the APACHE 2 Score and ICU and hospital days, there were no significant differences between the groups. Significant differences were found in age, SAPS 2, ventilation days, ICU and hospital mortality, all of which scored items and points in the dynamic scoring system (Table 1). Analysis of the inflammatory parameters did not show any correlation between the score groups, apart from patients with a DSS $<6$ points had the lowest PCT levels.

In the overall patient population, the primary endpoint analysis showed that higher DSS scores were associated with an increase in day 56 mortality (<6 vs. $>8 ; p=0.004)$ (Figure 2), when both ICU and hospital mortality are considered. Kaplan-Meier curves showing the effect at day 56 as well as day 7, are provided below (Figures 2 and 3). 
Table 1. Baseline characteristics, DSS relevant parameters and outcome variables depending on score groups in the overall patient cohort. Presented are mean values \pm standard deviations, frequency and percent $(\%)$ and levels of significance.

\begin{tabular}{|c|c|c|c|c|c|c|}
\hline & $\begin{array}{l}\text { DSS }<6 \\
(n=98)\end{array}$ & $\begin{array}{l}\text { DSS 6-8 } \\
(n=294)\end{array}$ & $\begin{array}{l}\text { DSS > 8 } \\
(n=110)\end{array}$ & $\begin{array}{c}p \text {-Value } \\
\text { (DSS < 6 vs. } \\
\text { DSS 6-8) }\end{array}$ & $\begin{array}{c}p \text {-Value } \\
\text { (DSS < } 6 \text { vs. } \\
\text { DSS > 8) }\end{array}$ & $\begin{array}{c}p \text {-Value } \\
\text { (DSS 6-8 vs. } \\
\text { DSS > 8) }\end{array}$ \\
\hline Age (years) & $68.5( \pm 12.4)$ & $65.11( \pm 14.7)$ & $66.6( \pm 14.4)$ & 0.027 & 0.325 & 0.335 \\
\hline APACHE II (points) & $35.6( \pm 9.2)$ & $36.1( \pm 10.2)$ & $36.9( \pm 9.5)$ & 0.617 & 0.321 & 0.515 \\
\hline SAPS II (points) & $50.2( \pm 13.2)$ & $53.7( \pm 14.8)$ & $62.3( \pm 18.6)$ & 0.033 & $<0.001 *$ & $<0.001 *$ \\
\hline Ventilator days & $7.8( \pm 10.1)$ & $11.1( \pm 11.1)$ & $11.7( \pm 16.1)$ & $0.016^{*}$ & 0.038 & 0.776 \\
\hline ICU stay (days) & $13.2( \pm 11.9)$ & $17.2( \pm 21.0)$ & $16.4( \pm 19.8)$ & 0.073 & 0.173 & 0.695 \\
\hline Hospital stay (days) & $24.1( \pm 24.1)$ & $26.3( \pm 31.0)$ & $23.1( \pm 34.0)$ & 0.457 & 0.804 & 0.379 \\
\hline ICU mortality $(\%)$ & $42(42.9 \%)$ & $165(56.1 \%)$ & $71(64.5 \%)$ & 0.023 & $0.002 *$ & 0.127 \\
\hline Hospital mortality (\%) & $48(49.0 \%)$ & $173(58.8 \%)$ & $79(71.8 \%)$ & 0.089 & $<0.001 *$ & $0.017 *$ \\
\hline Lactate $\mathrm{T}_{0}(\mathrm{mmol} / \mathrm{L})$ & $3.90( \pm 3.61)$ & $4.65( \pm 3.50)$ & $4.93( \pm 3.21)$ & 0.074 & 0.032 & 0.450 \\
\hline Lactate $\mathrm{T}_{6}(\mathrm{mmol} / \mathrm{L})$ & $2.65( \pm 2.89)$ & $3.98( \pm 3.25)$ & $6.74( \pm 3.85)$ & $<0.001 *$ & $<0.001 *$ & $<0.001 *$ \\
\hline $\begin{array}{l}\text { Norepinephrine } T_{0} \\
(\mu \mathrm{g} / \mathrm{kg} / \mathrm{min})\end{array}$ & $0.21( \pm 0.25)$ & $0.39( \pm 0.41)$ & $0.43( \pm 0.42)$ & $<0.001$ * & $<0.001$ * & 0.314 \\
\hline $\begin{array}{l}\text { Norepinephrine } \mathrm{T}_{6} \\
(\mu \mathrm{g} / \mathrm{kg} / \mathrm{min})\end{array}$ & $0.20( \pm 0.19)$ & $0.49( \pm 0.40)$ & $0.72( \pm 0.36)$ & $<0.001$ * & $<0.001 *$ & $<0.001$ * \\
\hline Second catecholamine $\mathrm{T}_{0}(\%)$ & $3(3.1 \%)$ & $81(27.6 \%)$ & $68(61.8 \%)$ & $<0.001 *$ & $<0.001 *$ & $<0.001 *$ \\
\hline Hydrocortisone $\mathrm{T}_{0}(\%)$ & $5(5.1 \%)$ & $135(45.9 \%)$ & $89(80.9 \%)$ & $<0.001 *$ & $<0.001 *$ & $<0.001 *$ \\
\hline Volume bolus used (mL/kg) & $62.4( \pm 20.4)$ & $80.4( \pm 28.8)$ & $90.0( \pm 31.8)$ & $<0.001 *$ & $<0.001 *$ & $0.006^{*}$ \\
\hline $\begin{array}{c}\text { Dynamic Scoring System } \\
\text { (points) }\end{array}$ & $4.41( \pm 0.94)$ & $7.04( \pm 0.81)$ & $9.63( \pm 0.77)$ & $<0.001$ * & $<0.001$ * & $<0.001$ * \\
\hline
\end{tabular}

* = Statistically significant using Bonferroni-adjusted alpha critical value $=0.017$

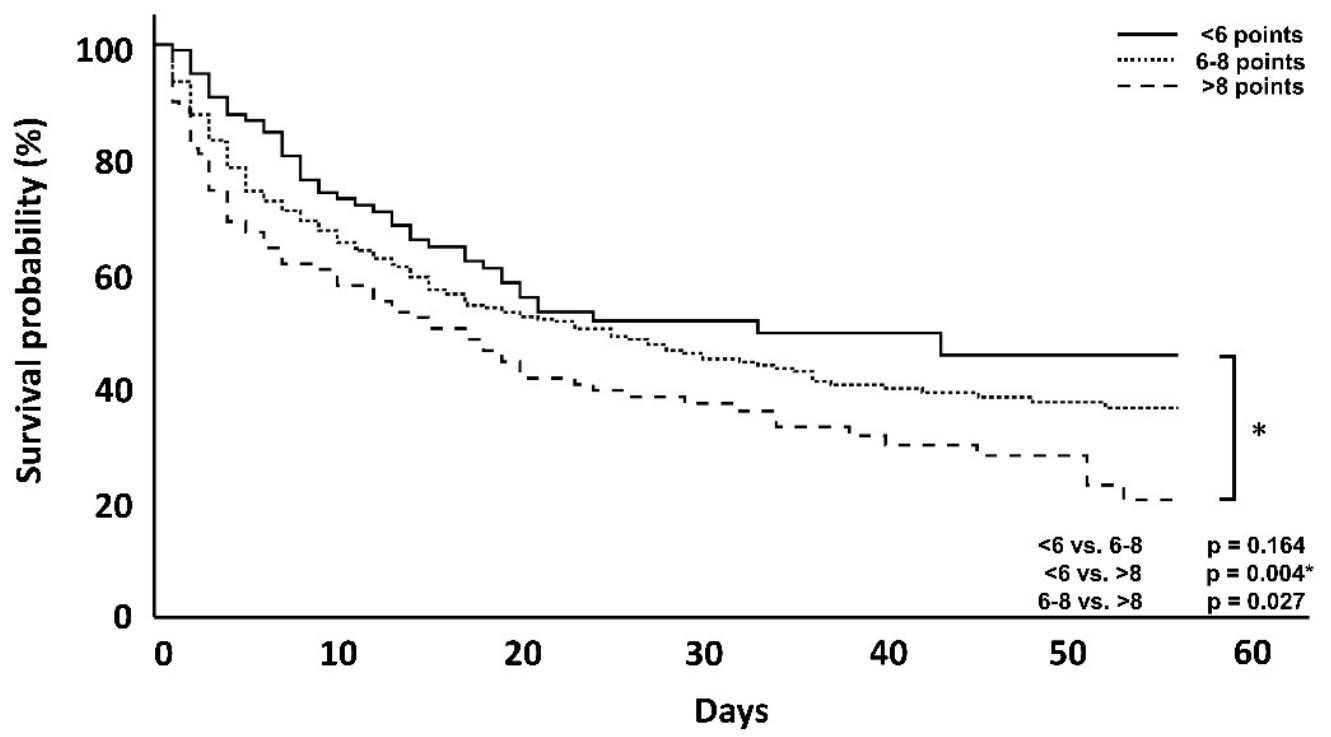

Figure 2. Kaplan-Meyer survival analysis at day 56 of the entire patient cohort. The $p$-values are via log-rank tests. ${ }^{*}=$ Statistically significant using Bonferroni-adjusted alpha critical value $=0.017$ for groups $<6$ vs. $>8$, but no other comparisons. 


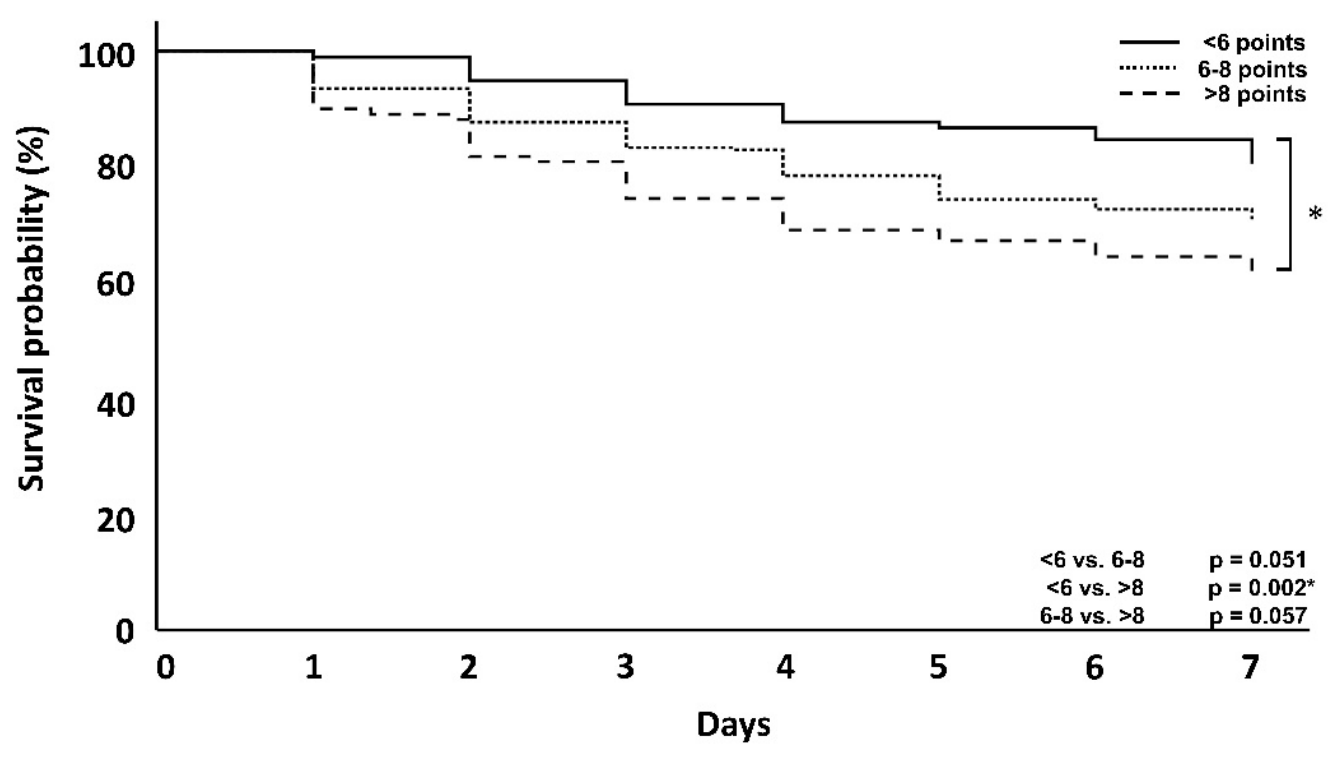

Figure 3. Kaplan-Meyer survival analysis at day 7 of the entire patient cohort. The $p=$ value is via log-rank test. ${ }^{*}=$ Statistically significant using Bonferroni-adjusted alpha critical value $=0.017$ for groups $<6$ vs. $>8$, but no other comparisons.

A total of 198 patients were treated with CRRT and CytoSorb, 61.1\% of whom were male. The diagnoses in this study population included pneumonia $(n=76,38.3 \%)$, abdominal sepsis $(n=85,42.9 \%)$, urosepsis $(n=13,6.5 \%)$ and miscellaneous $(n=24,12.1 \%)$. The baseline characteristics are depicted in Table 2 . With regard to gender, the APACHE 2 Score and ICU and hospital days as well as ICU-mortality, there were no differences between the groups. Significant differences were found in age, SAPS 2, ventilation days, hospital mortality and time delay until start of therapy, all of which scored items without $\mathrm{T}_{0}$ lactate and points in the dynamic scoring system (Table 2).

Table 2. Baseline characteristics, DSS relevant parameters and outcome variables depending on score groups in the CytoSorb group. Presented are mean values \pm standard deviations, frequency and percent $(\%)$ and levels of significance.

\begin{tabular}{|c|c|c|c|c|c|c|}
\hline & $\begin{array}{l}\text { DSS }<6 \\
(n=17)\end{array}$ & $\begin{array}{l}\text { DSS 6-8 } \\
(n=118)\end{array}$ & $\begin{array}{l}\text { DSS }>8 \\
(n=63)\end{array}$ & $\begin{array}{c}p \text {-Value } \\
\text { (DSS < } 6 \text { vs. } \\
\text { DSS 6-8) }\end{array}$ & $\begin{array}{c}p \text {-Value } \\
\text { (DSS < } 6 \text { vs. } \\
\text { DSS > 8) }\end{array}$ & $\begin{array}{c}p \text {-Value } \\
\text { (DSS 6-8 vs. } \\
\text { DSS > 8) }\end{array}$ \\
\hline Age (years) & $66.8( \pm 10.43)$ & $60.5( \pm 14.80)$ & $64.4( \pm 15.76)$ & 0.037 & 0.462 & 0.097 \\
\hline APACHE II (points) & $34.0( \pm 9.29)$ & $33.4( \pm 10.23)$ & $34.6( \pm 10.03)$ & 0.811 & 0.838 & 0.473 \\
\hline SAPS II (points) & $56.2( \pm 18.81)$ & $56.6( \pm 15.30)$ & $65.8( \pm 19.85)$ & 0.947 & 0.079 & $<0.001 *$ \\
\hline Ventilator days & $7.6( \pm 8.17)$ & $13.9( \pm 19.05)$ & $13.0( \pm 18.53)$ & 0.022 & 0.247 & 0.773 \\
\hline ICU stay (days) & $12.4( \pm 8.20)$ & $21.4( \pm 25.20)$ & $17.9( \pm 22.85)$ & 0.147 & 0.123 & 0.355 \\
\hline Hospital stay (days) & $26.1( \pm 33.70)$ & $30.9( \pm 37.39)$ & $25.35( \pm 40.39)$ & 0.599 & 0.939 & 0.356 \\
\hline ICU mortality (\%) & $11(64.7 \%)$ & $66(55.9 \%)$ & $41(65.1 \%)$ & 0.501 & 0.978 & 0.235 \\
\hline Hospital mortality (\%) & $13(76.5 \%)$ & $67(56.8 \%)$ & $46(73.0 \%)$ & 0.122 & 0.774 & 0.032 \\
\hline $\begin{array}{l}\text { CytoSorb therapy delay } \\
\text { (hours) }\end{array}$ & $52.6( \pm 30.50)$ & $23.0( \pm 21.50)$ & $18.20( \pm 20.57)$ & $<0.001$ * & $<0.001$ * & 0.138 \\
\hline $\begin{array}{l}\text { Number of CytoSorb } \\
\text { adsorbers used (n) }\end{array}$ & $2.2( \pm 0.77)$ & $2.7( \pm 1.57)$ & $2.7( \pm 1.58)$ & 0.230 & 0.117 & 0.895 \\
\hline Lactate $\mathrm{T}_{0}(\mathrm{mmol} / \mathrm{L})$ & $3.12( \pm 3.49)$ & $4.87( \pm 3.81)$ & $5.01( \pm 3.26)$ & 0.076 & 0.041 & 0.805 \\
\hline
\end{tabular}


Table 2. Cont.

\begin{tabular}{|c|c|c|c|c|c|c|}
\hline & $\begin{array}{l}\text { DSS <6 } \\
(n=17)\end{array}$ & $\begin{array}{l}\text { DSS 6-8 } \\
(n=118)\end{array}$ & $\begin{array}{l}\text { DSS > 8 } \\
(n=63)\end{array}$ & $\begin{array}{c}p \text {-Value } \\
\text { (DSS < } 6 \text { vs. } \\
\text { DSS 6-8) }\end{array}$ & $\begin{array}{c}p \text {-Value } \\
\text { (DSS < } 6 \text { vs. } \\
\text { DSS > 8) }\end{array}$ & $\begin{array}{c}p \text {-Value } \\
\text { (DSS 6-8 vs. } \\
\text { DSS > 8) }\end{array}$ \\
\hline Lactate $\mathrm{T}_{6}(\mathrm{mmol} / \mathrm{L})$ & $2.40( \pm 3.02)$ & $4.09( \pm 3.34)$ & $6.60( \pm 3.17)$ & 0.051 & $<0.001 *$ & $<0.001$ * \\
\hline $\begin{array}{l}\text { Norepinephrine } \mathrm{T}_{0} \\
(\mu \mathrm{g} / \mathrm{kg} / \mathrm{min})\end{array}$ & $0.31( \pm 0.26)$ & $0.48( \pm 0.50)$ & $0.47( \pm 0.46)$ & 0.036 & 0.169 & 0.906 \\
\hline $\begin{array}{c}\text { Norepinephrine } \mathrm{T}_{6} \\
(\mu \mathrm{g} / \mathrm{kg} / \mathrm{min})\end{array}$ & $0.31( \pm 0.25)$ & $0.50( \pm 0.42)$ & $0.75( \pm 0.35)$ & $0.012 *$ & $<0.001 *$ & $<0.001$ * \\
\hline Second catecholamine $\mathrm{T}_{0}(\%)$ & $3(17.6 \%)$ & $57(48.3 \%)$ & $51(81.0 \%)$ & $0.017^{*}$ & $<0.001 *$ & $<0.001 *$ \\
\hline Hydrocortisone $\mathrm{T}_{0}(\%)$ & $4(23.5 \%)$ & $78(66.1 \%)$ & $54(85.7 \%)$ & $<0.001 *$ & $<0.001$ * & $0.003 *$ \\
\hline Volume bolus used (mL/kg) & $63.0( \pm 14.4)$ & $77.4( \pm 27.0)$ & $82.8( \pm 28.8)$ & 0.038 & 0.011 * & 0.261 \\
\hline $\begin{array}{l}\text { Dynamic Scoring System } \\
\text { (points) }\end{array}$ & $4.23( \pm 0.97)$ & $7.22( \pm 0.82)$ & $9.84( \pm 0.86)$ & $<0.001$ * & $<0.001$ * & $<0.001$ * \\
\hline
\end{tabular}

$*=$ Statistically significant using Bonferroni-adjusted alpha critical value $=0.017$.

In this cohort of patients, who received CytoSorb treatment, those with a DSS $<6$ points counterintuitively showed an increased ICU and hospital mortality when compared with clusters with 6-8 points. However, a comparison of the clusters with 6-8 points and $>8$ points again confirmed the trend of higher DSS scorings being linked to an increased mortality. Taking a closer look at the first 7 days, however, patients with a score $<6$ showed, as originally expected, a lower mortality compared to the other groups ( 23.5 vs. 24.4 vs. $38.7 \%$ ) (Table 3). Therefore, apart from the above-mentioned exceptions, differences in mortality showed a trend with increasing score groups in the first 7 days, after 56 days and also with regard to ICU and hospital mortality (Table 2, Figures 4 and 5).

Table 3. Mortality rates in different patient groups. Presented are frequencies, percentages and levels of significance.

\begin{tabular}{|c|c|c|c|c|c|c|}
\hline & $\begin{array}{l}\text { All Patients, } \\
\text { DSS < } 6\end{array}$ & $\begin{array}{l}\text { All Patients, } \\
\text { DSS 6-8 }\end{array}$ & $\begin{array}{l}\text { All Patients, } \\
\text { DSS > } 8\end{array}$ & $\begin{array}{c}p \text {-Value } \\
(<6 \text { vs. } 6-8)\end{array}$ & $\begin{array}{c}p \text {-Value } \\
(<6 \text { vs. }>8)\end{array}$ & $\begin{array}{c}p \text {-Value } \\
(6-8 \text { vs. }>8)\end{array}$ \\
\hline 7-day mortality & $19(19.4 \%)$ & $85(28.9 \%)$ & $42(38.2 \%)$ & 0.051 & $0.002 *$ & 0.057 \\
\hline 56-day mortality & $45(45.9 \%)$ & $165(56.1 \%)$ & $76(69.1 \%)$ & 0.164 & 0.004 * & 0.027 \\
\hline ICU mortality & $42(42.8 \%)$ & $165(56.1 \%)$ & $71(64.5 \%)$ & 0.023 & $0.002 *$ & 0.127 \\
\hline \multirow[t]{2}{*}{ Hospital mortality } & $48(48.9 \%)$ & $173(58.8 \%)$ & $80(72.7 \%)$ & 0.089 & $<0.001 *$ & 0.017 * \\
\hline & $\begin{array}{l}\text { CytoSorb } \\
\text { Patients, } \\
\text { DSS < } 6\end{array}$ & $\begin{array}{l}\text { CytoSorb } \\
\text { Patients, } \\
\text { DSS 6-8 }\end{array}$ & $\begin{array}{l}\text { CytoSorb } \\
\text { Patients, } \\
\text { DSS > } 8\end{array}$ & $\begin{array}{c}p \text {-Value } \\
(<6 \text { vs. } 6-8)\end{array}$ & $\begin{array}{c}p \text {-Value } \\
(<6 \text { vs. }>8)\end{array}$ & $\begin{array}{c}p \text {-Value } \\
(6-8 \text { vs. }>8)\end{array}$ \\
\hline 7-day mortality & $4(23.5 \%)$ & $29(24.4 \%)$ & $24(38.7 \%)$ & 0.850 & 0.030 & 0.191 \\
\hline 56-day mortality & $12(70.5 \%)$ & $66(55.5 \%)$ & $44(71.0 \%)$ & 0.269 & 0.040 & 0.805 \\
\hline ICU mortality & $11(64.7 \%)$ & $66(55.9 \%)$ & $41(65.0 \%)$ & 0.501 & 0.978 & 0.235 \\
\hline \multirow[t]{2}{*}{ Hospital mortality } & $13(76.4 \%)$ & $67(56.7 \%)$ & $47(74.6 \%)$ & 0.102 & 0.877 & 0.018 \\
\hline & $\begin{array}{c}\text { CytoSorb } \\
\text { Therapy } \\
\text { Delay } \leq 12 \mathrm{~h}\end{array}$ & $\begin{array}{c}\text { CytoSorb } \\
\text { Therapy } \\
\text { Delay }>12-24 \mathrm{~h}\end{array}$ & $\begin{array}{c}\text { CytoSorb } \\
\text { Therapy } \\
\text { Delay }>24 \text { h }\end{array}$ & $\begin{array}{l}p \text {-Value } \\
(\leq 12 \text { vs. } \\
>12-24 \text { h })\end{array}$ & $\begin{array}{l}p \text {-Value } \\
(\leq 12 \text { vs. } \\
>24 \mathrm{~h})\end{array}$ & $\begin{array}{c}p \text {-Value } \\
(>12-24 \text { vs } \\
>24 \text { h) }\end{array}$ \\
\hline 7-day mortality & $23(29.1 \%)$ & $21(31.8 \%)$ & $18(34.0 \%)$ & 0.755 & 0.607 & 0.836 \\
\hline 56-day mortality & $44(55.7 \%)$ & $39(59.1 \%)$ & $39(73.6 \%)$ & 0.795 & 0.037 & 0.073 \\
\hline ICU mortality & $40(50.0 \%)$ & $39(60.0 \%)$ & $39(73.5 \%)$ & 0.231 & $0.006^{*}$ & 0.123 \\
\hline Hospital mortality & $45(56.2 \%)$ & $40(61.5 \%)$ & $42(79.2 \%)$ & 0.430 & $0.006^{*}$ & 0.038 \\
\hline
\end{tabular}




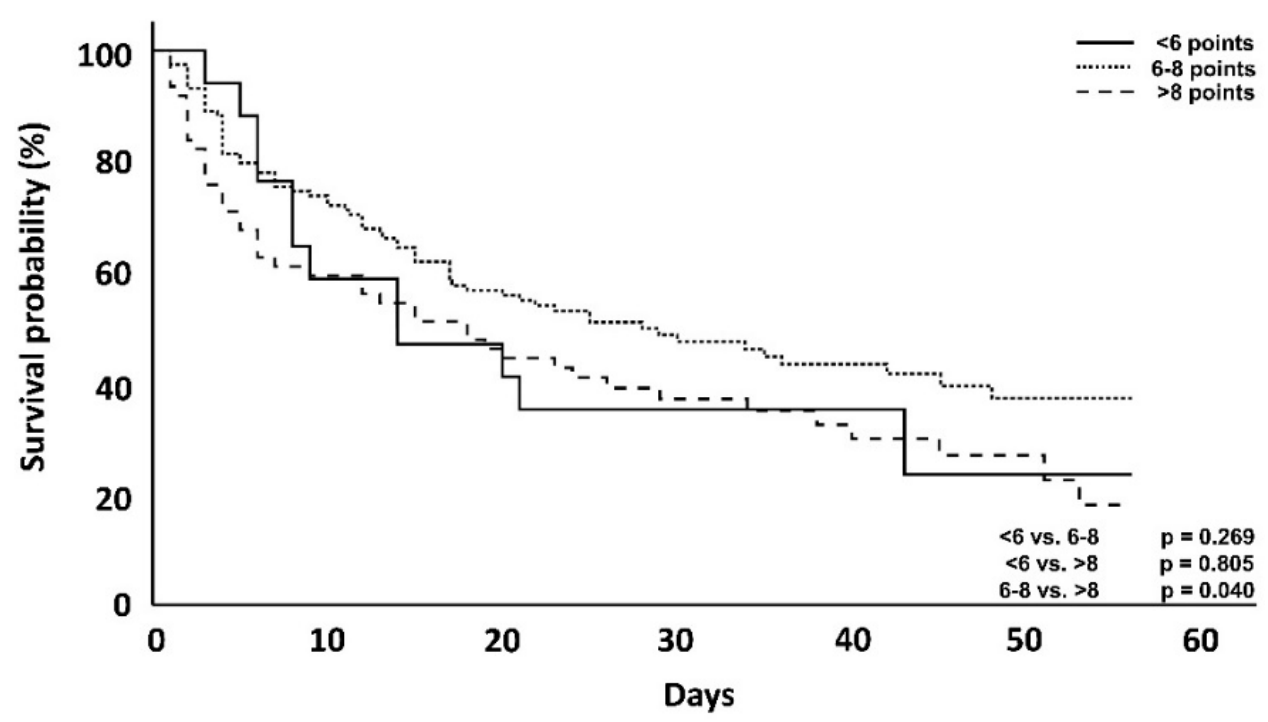

Figure 4. Kaplan-Meyer survival analysis at day 56 of CytoSorb treated patients. The $p$-value is via log-rank test.

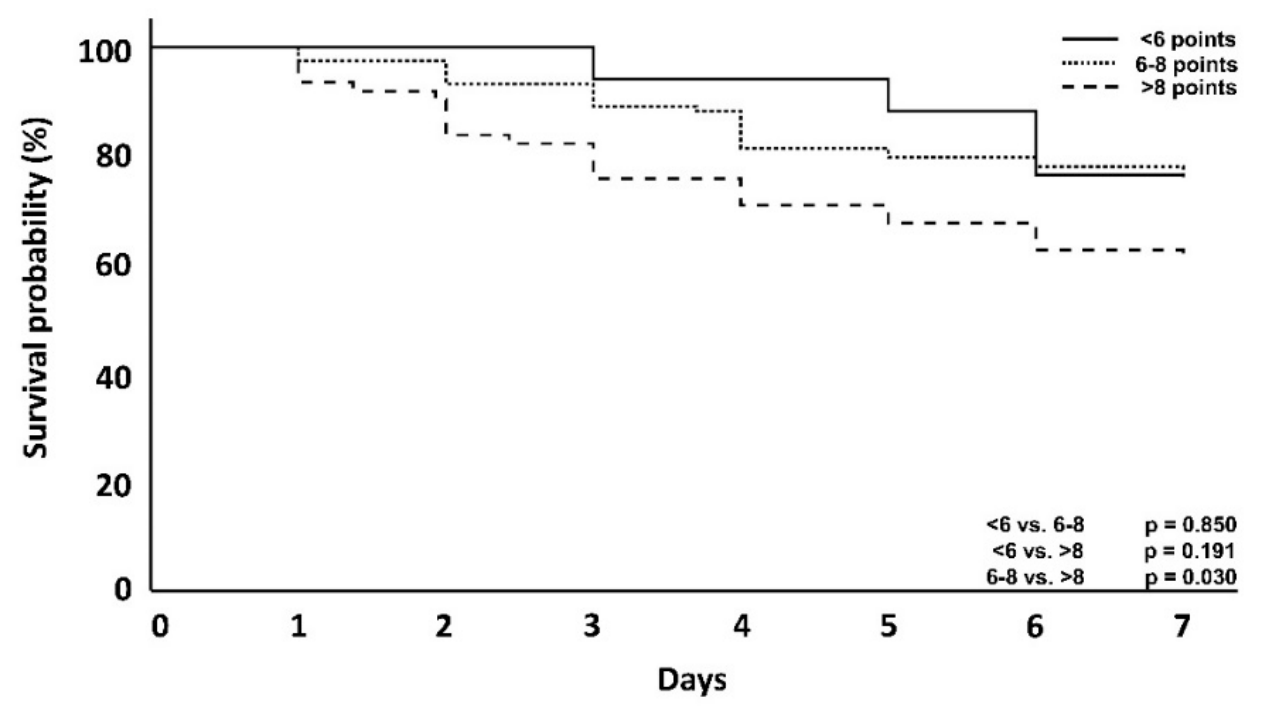

Figure 5. Kaplan-Meyer survival analysis at day 7 of CytoSorb treated patients. The $p$-value is via log-rank test.

To also investigate the association between mortality and the time until the start of hemoadsorption therapy in patients receiving CytoSorb, the delay from onset of septic shock $\left(\mathrm{T}_{0}\right)$ to the initiation of hemoadsorption therapy was clustered into different time intervals ( $\leq 12 \mathrm{~h},>12-24 \mathrm{~h},>24 \mathrm{~h}$ ). The time until the start of therapy correlated positively with ICU and hospital mortality (Table 4). After 7 days, however, this was not significant (29.1 vs. 31.8 vs. 34\%), (Figure 6). After 56 days, mortality in the group with a 12-h delay was lower than in the group with $>24$-hour delay (Figure 7). 
Table 4. Baseline characteristics, DSS relevant parameters and outcome variables depending on therapy delays in the CytoSorb group. Presented are mean values \pm standard deviations, frequency and percent $(\%)$ and levels of significance.

\begin{tabular}{|c|c|c|c|c|c|c|}
\hline & $\begin{array}{c}\text { Therapy } \\
\text { Delay } \\
\leq 12 \mathrm{~h}(n=80)\end{array}$ & $\begin{array}{c}\text { Therapy Delay } \\
>12-24 \mathrm{~h} \\
(n=65)\end{array}$ & $\begin{array}{c}\text { Therapy } \\
\text { Delay } \\
>24 \text { h }(n=53) \\
\end{array}$ & $\begin{array}{l}p \text {-Value } \\
(<12 \text { vs. } \\
12-24 \text { h) }\end{array}$ & $\begin{array}{c}p \text {-Value } \\
\text { (<12 vs. } \\
>24 \mathrm{~h} \text { ) }\end{array}$ & $\begin{array}{c}p \text {-Value } \\
(12-24 \text { vs. } \\
>24 \mathrm{~h})\end{array}$ \\
\hline Age (years) & $62.0( \pm 13.72)$ & $61.2( \pm 16.06)$ & $64.1( \pm 15.28)$ & 0.670 & 0.410 & 0.310 \\
\hline APACHE II (points) & $32.7( \pm 9.38)$ & $36.2( \pm 9.31)$ & $32.7( \pm 11.53)$ & 0.028 & 0.994 & 0.078 \\
\hline SAPS II (points) & $64.2( \pm 19.26)$ & $56.1( \pm 14.93)$ & $56.7( \pm 16.96)$ & $0.007^{*}$ & 0.021 & 0.850 \\
\hline Ventilator days & $12.9( \pm 18.50)$ & $13.2( \pm 19.11)$ & $13.0( \pm 16.93)$ & 0.930 & 0.996 & 0.937 \\
\hline ICU stay (days) & $20.2( \pm 25.80)$ & $19.9( \pm 23.95)$ & $18.0( \pm 19.49)$ & 0.930 & 0.578 & 0.643 \\
\hline Hospital stay (days) & $28.1( \pm 39.99)$ & $32.1( \pm 42.71)$ & $25.0( \pm 29.98)$ & 0.560 & 0.609 & 0.293 \\
\hline ICU mortality (\%) & $40(50.0 \%)$ & $39(60.0 \%)$ & $39(73.5 \%)$ & 0.231 & $0.006^{*}$ & 0.123 \\
\hline Hospital mortality (\%) & $45(56.2 \%)$ & $40(61.5 \%)$ & $42(79.2 \%)$ & 0.430 & $0.006^{*}$ & 0.038 \\
\hline CytoSorb therapy delay (hours) & $6.8( \pm 4.50)$ & $20.4( \pm 3.99)$ & $54.6( \pm 25.92)$ & $<0.001 *$ & $<0.001$ * & $<0.001 *$ \\
\hline Number of CytoSorb adsorbers used (n) & $3.03( \pm 1.66)$ & $2.69( \pm 1.62)$ & $2.24( \pm 0.97)$ & 0.211 & $0.002 *$ & 0.082 \\
\hline Lactate $\mathrm{T}_{0}(\mathrm{mmol} / \mathrm{L})$ & $4.73( \pm 3.48)$ & $5.11( \pm 3.90)$ & $4.38( \pm 3.55)$ & 0.540 & 0.574 & 0.292 \\
\hline Lactate $\mathrm{T}_{6}(\mathrm{mmol} / \mathrm{L})$ & $5.09( \pm 3.63)$ & $5.05( \pm 3.67)$ & $3.80( \pm 3.00)$ & 0.940 & 0.034 & 0.046 \\
\hline Norepinephrine $\mathrm{T}_{0}(\mu \mathrm{g} / \mathrm{kg} / \mathrm{min})$ & $0.50( \pm 0.65)$ & $0.45( \pm 0.26)$ & $0.42( \pm 0.37)$ & 0.506 & 0.323 & 0.613 \\
\hline Norepinephrine $\mathrm{T}_{6}(\mu \mathrm{g} / \mathrm{kg} / \mathrm{min})$ & $0.64( \pm 0.46)$ & $0.54( \pm 0.35)$ & $0.50( \pm 0.40)$ & 0.153 & 0.083 & 0.647 \\
\hline Second catecholamine $\mathrm{T}_{0}(\%)$ & $64(80.0 \%)$ & $25(38.0 \%)$ & $22(41.5 \%)$ & $<0.001 *$ & $<0.001 *$ & 0.740 \\
\hline Hydrocortisone $\mathrm{T}_{0}(\%)$ & $63(78.7 \%)$ & $44(67.6 \%)$ & $29(56.6 \%)$ & 0.134 & $0.008 *$ & 0.235 \\
\hline Volume bolus used $(\mathrm{mL} / \mathrm{kg})$ & $75.0( \pm 25.8)$ & $81.6( \pm 28.8)$ & $78.0( \pm 27.6)$ & 0.122 & 0.510 & 0.444 \\
\hline Dynamic Scoring System (points) & $8.48( \pm 1.53)$ & $7.60( \pm 1.58)$ & $7.01( \pm 2.19)$ & 0.001 * & $<0.001$ * & 0.093 \\
\hline
\end{tabular}

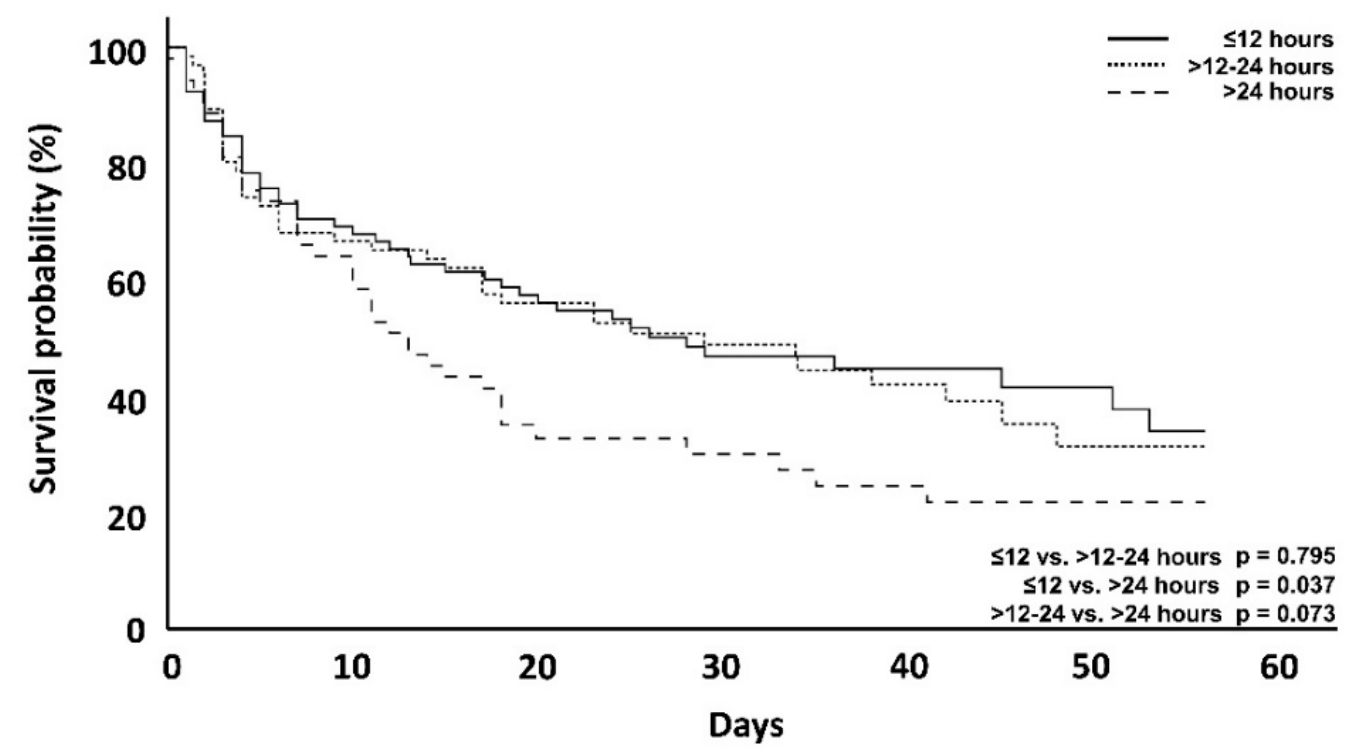

Figure 6. Kaplan-Meyer survival analysis of CytoSorb treated patients at day 56 with regard to different delay groups. The $p$-value is via log-rank test. 


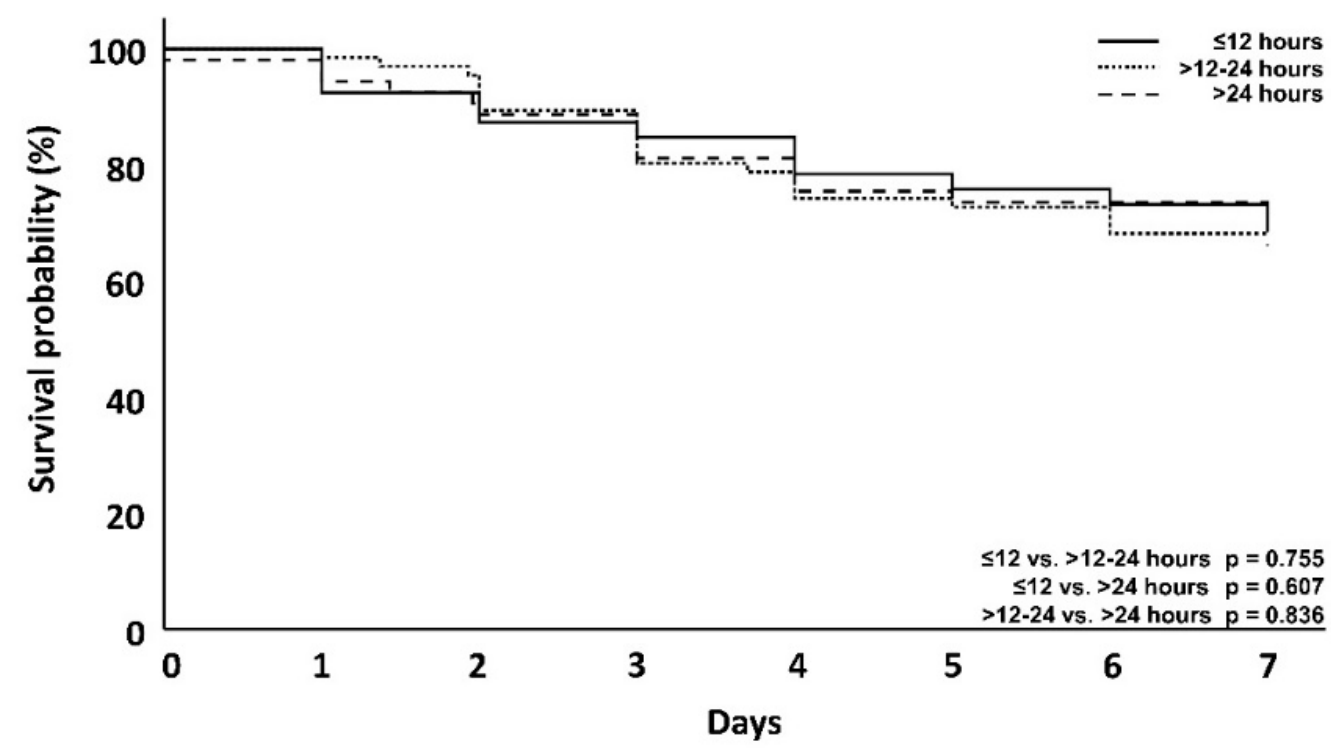

Figure 7. Kaplan-Meyer survival analysis of CytoSorb treated patients at day 7 with regard to different delay groups. The $p$-value is via Log-rank test.

Mortality was lowest in the patients where therapy was started early, despite the patients having higher lactate levels and norepinephrine needs and a higher need for a second catecholamine and/or hydrocortisone. This was significant for ICU mortality at $\leq 12$ vs. $>24 \mathrm{~h}$ therapy delay (50 vs. $73.5 \%, p=0.006$ ), for hospital mortality when comparing $\leq 12$ vs. $>24 \mathrm{~h}$ therapy delay (55 vs. $79.2 \%, p=0.004$ ) and for $12-24 \mathrm{~h}$ vs. $>24 \mathrm{~h}$ therapy delay (61.5 vs. $79.2 \%, p=0.038$ ) (Table 3$)$.

The mean therapy delay for the defined time intervals was significantly different (6.8, 20.4 and $54.6 \mathrm{~h}$, respectively; $p<0.001)$. The patients in whom therapy was started earliest, had the highest DSS score ( 8.4 vs. 7.6 vs. 7.0 DSS points), while patients with a low DSS had a longer delay until the start of CytoSorb (Tables 2 and 4). For better comparability of patients according to their severity of illness, we analyzed the subgroup of septic patients who did not receive CytoSorb therapy but who had ARF with a need for CRRT $(n=69)$, especially as all the CytoSorb-treated patients had also received concomitant CRRT due to ARF. The baseline characteristics are depicted in Table 5. With regard to gender, APACHE 2 Score, ventilation and ICU and hospital days, there were no differences between these patients in comparison with the CytoSorb patients group. ICU and hospital mortality in the CytoSorb-treated patients were lower, but not significant (Table 5).

In the CytoSorb group, a second catecholamine (56 vs. $24.6 \%$ ) as well as hydrocortisone (69.1 vs. 53.6\%) were used more frequently. The same held true for norepinephrine demand, which was also significantly increased at $\mathrm{T}_{0}$ and $\mathrm{T}_{6}$ in this group and, therefore, suggests that patients in the CytoSorb group had an increased disease severity. If therapy delay is combined with the disease severity (determined by the DSS score), the mortality of CytoSorb patients is lower in the group with 6-8 points and a therapy delay $\leq 12 \mathrm{~h}$, becoming significant in the group with $>8$ points (Table 6) for ICU mortality, after a Bonferroni adjustment. 
Table 5. Baseline characteristics, DSS relevant parameters and outcome variables in control + RRT patients and CytoSorb-treated individuals. Presented are mean values \pm standard deviations, frequency and percentage (\%) and levels of significance.

\begin{tabular}{|c|c|c|c|}
\hline & Control + RRT Group $(n=69)$ & CytoSorb Group $(n=198)$ & $p$-Value \\
\hline Age (years) & $66.2( \pm 12.4)$ & $62.3( \pm 14.9)$ & 0.035 \\
\hline APACHE II (points) & $39.8( \pm 9.6)$ & $33.8( \pm 10.0)$ & $<0.001$ \\
\hline SAPS II (points) & $56.2( \pm 14.8)$ & $59.5( \pm 17.6)$ & 0.144 \\
\hline Ventilator days & $12.3( \pm 13.4)$ & $13.0( \pm 18.2)$ & 0.715 \\
\hline ICU stay (days) & $19.9( \pm 18.1)$ & $19.5( \pm 23.5)$ & 0.882 \\
\hline Hospital stay (days) & $30.2( \pm 28.9)$ & $28.7( \pm 37.9)$ & 0.703 \\
\hline ICU mortality (\%) & $44(63.8 \%)$ & $118(59.6 \%)$ & 0.540 \\
\hline Hospital mortality (\%) & $49(71.0 \%)$ & $123(63.6 \%)$ & 0.268 \\
\hline Lactate $\mathrm{T}_{0}(\mathrm{mmol} / \mathrm{L})$ & $4.96( \pm 4.28)$ & $4.76( \pm 3.63)$ & 0.738 \\
\hline Lactate $\mathrm{T}_{6}(\mathrm{mmol} / \mathrm{L})$ & $5.08( \pm 4.40)$ & $4.73( \pm 3.52)$ & 0.565 \\
\hline Norepinephrine $\mathrm{T}_{0}(\mu \mathrm{g} / \mathrm{kg} / \mathrm{min})$ & $0.37( \pm 0.41)$ & $0.46( \pm 0.48)$ & 0.142 \\
\hline Norepinephrine $\mathrm{T}_{6}(\mu \mathrm{g} / \mathrm{kg} / \mathrm{min})$ & $0.55( \pm 0.44)$ & $0.57( \pm 0.41)$ & 0.783 \\
\hline Second catecholamine $\mathrm{T}_{0}(\%)$ & $17(24.6 \%)$ & $111(56.1 \%)$ & $<0.001$ \\
\hline Hydrocortisone $\mathrm{T}_{0}(\%)$ & $37(53.6 \%)$ & $136(68.7 \%)$ & 0.024 \\
\hline Volume bolus used (mL/kg) & $75.6( \pm 30.0)$ & $78.0( \pm 27.6)$ & 0.537 \\
\hline Dynamic Scoring System (points) & $7.20( \pm 1.68)$ & $7.80( \pm 1.82)$ & 0.014 \\
\hline
\end{tabular}

Table 6. Association between DSS score, therapy delay and outcome between control + RRT vs. CytoSorb group. Please note that groups containing not enough patients for a reliable statistical evaluation were classified as not applicable (n.a.); therefore, no analysis was performed in these groups. Presented are frequency and percentage (\%) and levels of significance.

\begin{tabular}{|c|c|c|c|c|c|c|}
\hline & \multicolumn{2}{|c|}{ ICU Mortality } & \multicolumn{4}{|c|}{ Hospital Mortality } \\
\hline & $\begin{array}{l}\text { Control + RRT } \\
\text { Group }(n=69)\end{array}$ & $\begin{array}{c}\text { CytoSorb } \\
\text { Group }(n=198)\end{array}$ & $p$-Value & $\begin{array}{l}\text { Control + RRT } \\
\text { Group }(n=69)\end{array}$ & $\begin{array}{c}\text { CytoSorb } \\
\text { Group }(n=198)\end{array}$ & $p$-Value \\
\hline $\begin{array}{c}\text { DSS }<6 \text { points, CytoSorb } \\
\text { delay } \leq 12 \mathrm{~h}\end{array}$ & n.a. & n.a. & n.a. & n.a. & n.a. & n.a. \\
\hline $\begin{array}{c}\text { DSS 6-8 points, CytoSorb } \\
\text { delay } \leq 12 \mathrm{~h}\end{array}$ & 27 (58.7\%) & $21(47.7 \%)$ & 0.303 & $30(65.2 \%)$ & $21(47.7 \%)$ & 0.096 \\
\hline $\begin{array}{c}\text { DSS }>8 \text { points, CytoSorb } \\
\text { delay } \leq 12 \mathrm{~h}\end{array}$ & $12(92.3 \%)$ & $17(51.5 \%)$ & 0.009 * & $12(92.3 \%)$ & $21(63.6 \%)$ & 0.053 \\
\hline $\begin{array}{c}\text { DSS }<6 \text { points, CytoSorb } \\
\text { delay }>12-24 \mathrm{~h}\end{array}$ & n.a. & n.a. & n.a. & n.a. & n.a. & n.a. \\
\hline $\begin{array}{c}\text { DSS 6-8 points, CytoSorb } \\
\text { delay }>12-24 \mathrm{~h}\end{array}$ & 27 (58.7\%) & $25(54.3 \%)$ & 0.678 & $30(65.2 \%)$ & $26(56.5 \%)$ & 0.398 \\
\hline $\begin{array}{c}\text { DSS }>8 \text { points, CytoSorb } \\
\text { delay }>12-24 \mathrm{~h}\end{array}$ & n.a. & n.a. & n.a. & n.a. & n.a. & n.a. \\
\hline $\begin{array}{c}\text { DSS }<6 \text { points, CytoSorb } \\
\text { delay }>24 \mathrm{~h}\end{array}$ & n.a. & n.a. & n.a. & n.a. & n.a. & n.a. \\
\hline $\begin{array}{c}\text { DSS 6-8 points, CytoSorb } \\
\text { delay }>24 \mathrm{~h}\end{array}$ & 27 (58.7\%) & $19(70.3 \%)$ & 0.318 & $30(65.2 \%)$ & 19 (70.3\%) & 0.654 \\
\hline $\begin{array}{c}\text { DSS }>8 \text { points, CytoSorb } \\
\text { delay }>24 \mathrm{~h}\end{array}$ & n.a. & n.a. & n.a. & n.a. & n.a. & n.a. \\
\hline
\end{tabular}

* = Statistically significant using Bonferroni-adjusted alpha critical value $=0.017$

A multivariate logistic regression model was fit to investigate an association between day 56 survival and selected predictor variables (Table 7). The results showed that the use of the CytoSorb device reduced the odds of mortality at day 56 by $44.8 \%$. With regard to the DSS Score, for each one unit increase in score, the odds of mortality at day 56 increased by $23.7 \%$ ( $p$ 0.001). Similarly, for each additional hour in CytoSorb therapy delay, the odds of mortality at day 56 increased by $1.5 \%(p=0.034)$; the associated use of renal 
replacement therapy (RRT) increased the odds of mortality at day 56 by $75.9 \%$; and lastly, for each one-year increase in patient age, the odds of mortality at day 56 increased by $3.7 \%$ $(p<0.001)$.

Table 7. Multivariate logistic regression results for predictors of day 56 survival.

\begin{tabular}{cccc}
\hline Predictor Variable & Odds Ratio & $\mathbf{9 5 \%}$ Confidence Interval & $p$-Value \\
\hline CytoSorb Therapy (Yes/No) & 0.552 & $0.275,1.108$ & 0.095 \\
DSS Score & 1.237 & $1.106,1.383$ & $<0.001$ \\
Therapy Delay (h) & 1.015 & $1.001,1.030$ & 0.034 \\
RRT (Yes/No) & 1.795 & $0.991,3.252$ & 0.054 \\
Age (Years) & 1.037 & $1.023,1.052$ & $<0.001$ \\
\hline
\end{tabular}

\section{Discussion}

In this retrospective, non-interventional, two-arm, multicentric data analysis, a newly created dynamic assessment system based on the clustering of established, clinically well-available and predominantly hemodynamics-associated parameters was used and evaluated in regard to its association with mortality. In the overall patient population (Table 1, Figures 2 and 3), a higher DSS score was associated with increased mortality at day 56, supporting the validity of the established procedure and analysis of data, but also the impact and predictivity of the assessed variables and their dynamic change on the outcome. In regard to the impact of the timing of hemoadsorption therapy, earlier initiation was shown to be associated with a better outcome.

With one exception, the correlation between higher scores and mortality was the same in the CytoSorb-treated patient population (Table 2, Figures 4 and 5), as CytoSorb treated patients with a DSS $<6$ failed to show this correlation. Similar findings were observed by Ferreira and colleagues in an analysis of the outcomes related to changes in the SOFA score [13]. The authors found an increased mortality in a group with a decrease in SOFA score points and presumed decreasing mortality, which was ultimately explained by the small number of patients (7.5\% of the total group). This was also exactly the case in our set of patients ( $n=17,8.5 \%$ of the total CytoSorb group). Moreover, taking into consideration the fact that timing seems to be a very important aspect of the therapy with impact on outcome, the delay time until the start of hemoadsorption therapy was longer in this small subgroup (52.6 h) compared to all other groups. Both can help to explain the higher mortality rate in these patients. In contrast to established scoring methods such as APACHE 2, SAPS 2 and SOFA, the score shown here does not describe a course over $24 \mathrm{~h}$ but can rather be used at the bedside within a very short time and, thus, describes early septic shock [13-16]. We performed survival analyses for the first 7 days and at day 56. Focusing on only the first 7 days in some analysis can be explained by the fact that the start of CytoSorb therapy is followed by an average of three days of therapy [17]; therefore, the maximum effect of this therapy is most likely to occur after day 3 and is potentially most pronounced in the first few days and not necessarily in the later clinical course $[4,5,7,9,10]$. However, there are also reports of differences in the outcome only observed at a later stage of the clinical course [18].

Several recent articles on the use of CytoSorb therapy have highlighted the potential benefits of an early start of hemoadsorption treatment $[4,5,7]$, which led us to analyze the correlation between the time delay until therapy initiation and mortality between the groups (Table 4, Figures 6 and 7). The mean therapy delay for the time intervals was significantly different between the DSS groups $(6.8,20.4$ and $54.6 \mathrm{~h}$, respectively; $p<0.001)$. A delayed initiation of hemoadsorption therapy was strongly associated with higher day-56, ICU and hospital mortality (Table 4), even though the patients in which CytoSorb therapy was started earlier, had higher norepinephrine requirements, an additional need for a second catecholamine as well as hydrocortisone and a higher DSS score (Table 2). Therefore, despite these patients being sicker than those treated later, they had a significantly better 
outcome, which further supports the assumption that timing as well as actual patient status are important criteria to consider in regard to the initiation of hemoadsorption therapy.

Acute renal failure in sepsis is common $[19,20]$ and associated with high mortality $[21,22]$. ARF in sepsis has a high mortality rate of more than $70 \%[23,24]$. A SepNet prevalence study from Germany showed a significantly higher mortality rate in septic patients with ARF compared to those without ARF (67.3 vs. $42.8 \%$ ) and concluded that ARF represents an independent risk factor for poor outcome in septic shock [25]. To shine a light on the role of acute renal failure and due to the fact that the CytoSorb-treated patients all received CRRT, we analyzed the subgroup of ECSISS patients, who did not receive CytoSorb therapy but who had also ARF with the need for CRRT $(n=69)$. Brouwer et al. showed that CytoSorb therapy was associated with a decreased observed versus expected 28-day allcause mortality and may be associated with a decreased all-cause mortality at 28 days compared to CRRT alone [26]. Recent results from Rugg et al. even showed an observed significant mortality difference between septic shock patients treated only with CRRT versus those treated with CRRT and adjunctive CytoSorb therapy [18]. Overall, our data suggest that the need for RRT is strongly associated with mortality (Tables 5 and 6 ). If the therapy delay for CytoSorb is analyzed together with the disease severity (determined by the DSS score) in regard to mortality and then compared to non-CytoSorb treated CRRT patients of the same DSS group, the mortality of CytoSorb patients with a therapy delay $\leq 12 \mathrm{~h}$ is lower in the DSS group of 6-8 points, becoming significant for ICU mortality in the group with $>8$ points (Table 6), a finding that further supports the recent results on the potential outcome benefits of CytoSorb therapy in patients with septic shock requiring CRRT. Finally, further analysis was performed to investigate the association of certain variables such as DSS score and therapy delay with mortality at day 56 via a multivariate logistic regression model (Table 7). The results support our initial findings and could even show some principle impacts of CytoSorb therapy on mortality, which, however, was not significant and also not the primary target of this investigation.

In our work, the severity of the disease in early septic shock is represented by the DSS score. Thus, more catecholamine requirements, the use of hydrocortisone and a second catecholamine as well as volume application and lactate plasma concentrations might represent disease severity better than 24-h scores such as APACHE 2, SOFA or SAPS 2 systemically can [13-16].

\section{Limitations}

This study was performed as a retrospective data collection and is not a prospective randomized, controlled trial. The evaluation may include patients in whom an escalation of therapy has been avoided. This is less likely in the CytoSorb group, since an additional procedure was already in place. There were a number of patients with a 'do not resuscitate' (DNR) order in the data sets, which were consequently not included. An influence of country- or clinic-specific therapy regimes cannot be ruled out. By weighting the therapy measures (application of volume, catecholamines, hydrocortisone; Figure 1) differently in the DSS matrix, we have tried to compensate for this. Last but not least, the created scoring system has yet to be validated in a prospective approach in regard to how it could help to identify patients that are likely to benefit from CytoSorb therapy in septic shock.

\section{Conclusions}

This newly created dynamic score allows for the assessment of hemodynamic development in the early phase of septic shock, thereby detecting the refractory status of septic patients and, finally, differentiating them into subgroups with different mortalities. This was given for all the patients examined and also for those who had received adjunctive CytoSorb therapy. Additionally, we could again show that the earlier CytoSorb therapy was started, the better the outcome was in terms of mortality. This easy-to-apply scoring system, which requiring only classical clinical hemodynamic information and one further laboratory value (lactate clearance), might present an option to better detect patients ben- 
efitting from the initiation of hemoadsorption therapy in septic shock, but prospective validation in this regard is required first.

Author Contributions: T.H., F.S., M.S. and D.J. were responsible for treatment of the patients and data recording. M.D. did the collection of the data and brought them into format including figures and tables and contributed to writing. K.K. was mainly responsible interpreting the data and for writing the manuscript. All authors have read and agreed to the published version of the manuscript.

Funding: There was no financial support for this study.

Institutional Review Board Statement: This study was approved by the ethics committee of the General Medical Council of Lower Saxony with reference number Bo/29/2019, followed by secondary ethics approvals for the other participating centres. The study was carried out according to the Declaration of Helsinki and in accordance with the Good Clinical Practice Protocol (GCP) (2001/20/EEC, CPMP/ICH/135/95), the established standard operating procedures and the local laws and regulations applicable to each country. Furthermore, the study was registered at ClinicalTrials.gov, accessed on 1 June 2021, (NCT03977688) before data retrieval and analysis started.

Informed Consent Statement: This study was approved by the ethics committee of the General Medical Council of Lower Saxony with reference number Bo/29/2019, followed by secondary ethics approvals for the other participating centres. The study was carried out according to the Declaration of Helsinki and in accordance with the Good Clinical Practice Protocol (GCP) (2001/20/EEC, $\mathrm{CPMP} / \mathrm{ICH} / 135 / 95)$, the established standard operating procedures and the local laws and regulations applicable to each country. Furthermore, the study was registered at ClinicalTrials.gov, accessed on 1 June 2021, (NCT03977688) before data retrieval and analysis started. Written informed consent was recommended by the ethics committee, however was not mandatory since data were analyzed in a pseudonymized fashion and as no intervention was involved. This is in accordance with the General Data Protection Regulation (GDPR). Although there is consent to the further use of clinical data in the hospital admission process in general, however this does not correspond to consent for study purposes.

Data Availability Statement: The datasets used and/or analyzed during the current study available from the corresponding author on reasonable request.

Acknowledgments: The authors would like to thank Peter Recknagel for his contributions in the preparation of the manuscript.

Conflicts of Interest: K.K., T.H., F.S. and M.D. received honoraria for lectures from CytoSorbents. The other authors have no conflicts of interest associated with this report.

\section{References}

1. Singer, M.; Deutschman, C.S.; Seymour, C.W.; Shankar-Hari, M.; Annane, D.; Bauer, M.; Bellomo, R.; Bernard, G.R.; Chiche, J.-D.; Coopersmith, C.M.; et al. The Third International Consensus Definitions for Sepsis and Septic Shock (Sepsis-3). JAMA 2016, 315, 801-810. [CrossRef]

2. Seymour, C.W.; Rosengart, M.R. Septic Shock: Advances in Diagnosis and Treatment. JAMA 2015, 314, 708-717. [CrossRef]

3. Fleischmann, C.; Thomas-Rueddel, D.O.; Hartmann, M.; Hartog, C.S.; Welte, T.; Heublein, S.; Dennler, U.; Reinhart, K. Hospital Incidence and Mortality Rates of Sepsis. Dtsch. Aerzteblatt Int. 2016, 113, 159-166. [CrossRef]

4. Kogelmann, K.; Jarczak, D.; Scheller, M.; Druner, M. Hemoadsorption by CytoSorb in septic patients-A case series. Crit. Care 2017, 21, 74. [CrossRef]

5. Friesecke, S.; Stecher, S.; Gross, S.; Felix, S.B.; Nierhaus, A. Extracorporeal Cytokine Elimination as Rescue Therapy in Refractory Septic Shock-A Prospective Single-Center Study. J. Artif. Organs 2017, 20, 252-259. [CrossRef] [PubMed]

6. Bassi, E.; Park, M.; Azevedo, L.C.P. Therapeutic Strategies for High-Dose Vasopressor-Dependent Shock. Crit. Care Res. Pract. 2013, 2013, 1-10. [CrossRef] [PubMed]

7. Hawchar, F.; László, I.; Öveges, N.; Trásy, D.; Ondrik, Z.; Molnar, Z. Extracorporeal cytokine adsorption in septic shock: A proof of concept randomized, controlled pilot study. J. Crit. Care 2019, 49, 172-178. [CrossRef]

8. Mehta, Y.; Mehta, C.; Kumar, A.; George, J.V.; Gupta, A.; Nanda, S.; Kochhar, G.; Raizada, A. Experience with hemoadsorption $\left(\mathrm{Cy}_{\text {-toSorb }}{ }^{\circledR}\right)$ in the management of septic shock patients. World J. Crit. Care Med. 2020, 9, 1-12. [CrossRef]

9. Träger, K.; Skrabal, C.; Fischer, G.; Datzmann, T.; Schroeder, J.; Fritzler, D.; Hartmann, J.; Liebold, A.; Reinelt, H. Hemoadsorption Treatment of Patients with Acute Infective Endocarditis during Surgery with Cardiopulmonary Bypass—A Case Series. Int. J. Artif. Organs 2017, 40, 240-249. [CrossRef] 
10. Leonardis, F.; De Angelis, V.; Frisardi, F.; Pietrafitta, C.; Riva, I.; Valetti, T.M.; Broletti, V.; Marchesi, G.; Menato, L.; Nani, R.; et al. Effect of Hemoadsorption for Cytokine Removal in Pneumococcal and Meningococcal Sepsis. Case Rep. Crit. Care 2018, $2018,1-7$. [CrossRef]

11. Rhodes, A.A.; Evans, L.E.; Alhazzani, W.; Levy, M.M.; Antonelli, M.; Ferrer, R.; Kumar, A.; Sevransky, J.E.; Sprung, C.L.; Nunnally, M.E.; et al. Surviving Sepsis Campaign: International Guidelines for Management of Sepsis and Septic Shock: 2016. Crit. Care Med. 2017, 45, 486-552. [CrossRef]

12. Vincent, J.L.; Moreno, R.; Takala, J.; Willatts, S.; De Mendonça, A.; Bruining, H.; Reinhart, C.K.; Suter, P.M.; Thijs, L.G. The SOFA (Sepsis-related Organ Failure Assessment) score to describe organ dysfunction/failure. On behalf of the Working Group on Sepsis-Related Problems of the European Society of Intensive Care Medicine. Intensive Care Med. 1996, 22, 707-710. [CrossRef] [PubMed]

13. Ferreira, F.L.; Bota, D.P.; Bross, A.; Melot, C.; Vincent, J.-L. Serial Evaluation of the SOFA Score to Predict Outcome in Critically Ill Patients. JAMA 2001, 286, 1754-1758. [CrossRef] [PubMed]

14. Lewandowski, K. Scoring-Systeme auf der Intensivtherapiestation. Der Anaesthesist 2003, 52, 965-990. [CrossRef]

15. Goldhill, D.R.; Withington, P.S. Mortality predicted by APACHE II. The effect of changes in physiological values and post-ICU hospital mortality. Anaesthesia 1996, 51, 719-723.

16. Knaus, W.A.; Draper, E.A.; Wagner, D.P.; Zimmerman, J.E. APACHE II: A severity of disease classification system. Crit. Care Med. 1985, 13, 818-829. [CrossRef]

17. Friesecke, S.; Träger, K.; Schittek, G.; Molnar, Z.; Bach, F.; Kogelmann, K.; Bogdanski, R.; Weyland, A.; Nierhaus, A.; Nestler, F.; et al. International registry on the use of the CytoSorb®adsorber in ICU patients: Study protocol and preliminary results. Med. Klin. Intensivmed. Notfallmed. 2019, 114, 699-707. [CrossRef] [PubMed]

18. Rugg, C.; Klose, R.; Hornung, R.; Innerhofer, N.; Bachler, M.; Schmid, S.; Fries, D.; Ströhle, M. Hemoadsorption with CytoSorb in Septic Shock reduces Catecholamine Requirements and In-Hospital Mortality: A Single-Center Retrospective 'Genetic' Matched Analysis. Biomedicines 2020, 8, 539. [CrossRef] [PubMed]

19. Bellomo, R.; Kellum, J.A.; Ronco, C.; Wald, R.; Martensson, J.; Maiden, M.; Bagshaw, S.M.; Glassford, N.J.; Lankadeva, Y.; Vaara, S.T.; et al. Acute kidney injury in sepsis. Intensive Care Med. 2017, 43, 816-828. [CrossRef] [PubMed]

20. Uchino, S.; Kellum, J.A.; Bellomo, R.; Doig, G.S.; Morimatsu, H.; Morgera, S.; Schetz, M.; Tan, I.; Bouman, C.; Macedo, E.; et al. Acute renal failure in critically ill patients: A multi-national, multicenter study. JAMA 2005, 294, 813-818. [CrossRef] [PubMed]

21. Khwaja, A. KDIGO Clinical Practice Guidelines for Acute Kidney Injury. Nephron 2012, 120, c179-c184. [CrossRef] [PubMed]

22. Barbar, S.D.; Clere-Jehl, R.; Bourredjem, A.; Hernu, R.; Montini, F.; Bruyère, R.; Lebert, C.; Bohé, J.; Badie, J.; Eraldi, J.-P.; et al. Timing of Renal-Replacement Therapy in Patients with Acute Kidney Injury and Sepsis. N. Engl. J. Med. 2018, 379, 1431-1442. [CrossRef] [PubMed]

23. Liaño, F.; Junco, E.; Pascual, J.; Madero, R.; Verde, E. The spectrum of acute renal failure in the intensive care unit compared with that seen in other settings. The Madrid Acute Renal Failure Study Group. Kidney Int. Suppl. 1998, 66, 16-24.

24. Chertow, G.M.; Burdick, E.; Honour, M.; Bonventre, J.V.; Bates, D.W. Acute kidney injury, mortality, length of stay, and costs in hos-pitalized patients. J. Am. Soc. Nephrol. 2005, 16, 3365-3370. [CrossRef]

25. Oppert, M.; Engel, C.; Brunkhorst, F.-M.; Bogatsch, H.; Reinhart, K.; Frei, U.; Eckardt, K.-U.; Loeffler, M.; John, S. German Competence Network Sepsis (Sepnet) Acute renal failure in patients with severe sepsis and septic shock-a significant independent risk factor for mortality: Results from the German Prevalence Study. Nephrol. Dial. Transplant. 2007, 23, 904-909. [CrossRef]

26. Brouwer, W.P.; Duran, S.; Kuijper, M.; Ince, C. Hemoadsorption with CytoSorb shows a decreased observed versus expected 28-day all-cause mortality in ICU patients with septic shock: A propensity-score-weighted retrospective study. Crit. Care 2019, 23, 1-9. [CrossRef] 